\title{
Intermédialités
}

Histoire et théorie des arts, des lettres et des techniques

Intermediality

History and Theory of the Arts, Literature and Technologies

\section{En débat : la reproduction numérique}

Numéro 17, printemps 2011

URI : https://id.erudit.org/iderudit/1005755ar

DOI : https://doi.org/10.7202/1005755ar

Aller au sommaire du numéro

Éditeur(s)

Revue intermédialités (Presses de l’Université de Montréal)

ISSN

1705-8546 (imprimé)

1920-3136 (numérique)

Découvrir la revue

Citer ce document

(2011). En débat : la reproduction numérique. Intermédialités / Intermediality, (17), 169-171. https://doi.org/10.7202/1005755ar d'utilisation que vous pouvez consulter en ligne.

https://apropos.erudit.org/fr/usagers/politique-dutilisation/ 


\section{En débat: \\ la reproduction numérique}

$N$ ous ouvrons ici un débat autour d'une question qui se situe au cœur même de l'intermédialité contemporaine: la reproduction numérique. Dans un numéro consacré aux différentes formes et techniques de reproduction, il nous a semblé important de soumettre à la discussion un texte de Bruno Latour et Adam Lowe propre à susciter la controverse dans la mesure où il défend l'exigence, pour que perdure une ouvre d'art, de la prolifération de ses reproductions. Selon les auteurs, la qualité d'une reproduction pourrait même surpasser celle de l'original en valeur «auratique». En partant de l'exemple de la duplication des Noces de Cana de Véronèse par le biais de techniques numériques et de son accrochage dans son lieu palladien d'origine à San Giorgio à Venise, ils envisagent ici la possibilité d'un détachement de l'aura par rapport à l'œuvre originale. . On sait que Walter Benjamin voyait l'aura annihilée par le besoin spécifiquement moderne de prendre possession immédiate de l'objet dans l'image et à travers ses reproductions ${ }^{2}$. Par voie de contraste, grâce aux nouvelles techniques de fac-similisation utilisant le numérique, l'aura pourrait pour Latour et Lowe s'attacher à l'une ou l'autre des reproductions. Ce processus dépendrait de la qualité de ce que l'on peut considérer être les différentes versions d'une ouvre ainsi que de la réussite de leur inscription dans un lieu particulier et de leur disponibilité au regard.

La réintégration d'un duplicata dans le lieu initial de l'ouvre, comme c'est le cas à San Giorgio, pourrait-elle réinstaurer la relation spatiale originelle entre la peinture de Véronèse et l'architecture dessinée par Palladio? Pensée ainsi, l'aura participerait-elle plus du lieu "intermédial» d'origine que de l'œuvre unique, arrachée de son contexte et déplacée dans un espace muséal? (Notons d'ailleurs que la duplication dans son espace premier de ce célèbre tableau a également inspiré

1. Ce texte est issu des Dialogues de San Giorgio «Inheriting the past. Tradition, translation, betrayal, innovation» organisés à Venise par la Fondazione Cini en septembre 2007 à la suite de l'accrochage du fac-similé des Noces de Cana de Véronèse dans son réfectoire palladien d'origine. Voir www.cini.it/en/event/detail/2/39 (dernière consultation le 22 juin 2011).

2. Voir Walter Benjamin, «L'œuvre d'art à l'époque de sa reproduction mécanisée », Écrits français, Paris, Gallimard, coll. «Bibliothèque des Idées », 1991, p. 183. 
à Peter Greenaway, in situ en 2009, l'une de ses grandes performances multimédiales ${ }^{3}$.) Le concept de reproduction qui semble visé par les auteurs s'attache plus à une pérennité esthétique des ouvres par le biais de la fac-similisation numérique qu'à explorer - comme le faisait Benjamin - les conséquences de la prolifération des images par les techniques de mécanisation sur la fonction même de l'art. Ainsi l'aura de l'œuvre serait-elle à l'ère du numérique devenue hésitante, ne sachant se décider quant au lieu où elle devrait se poser - un peu comme dans ces images pieuses où le Saint-Esprit, sous la forme d'une colombe lumineuse, venait parfois faire bénéficier les humains de sa divine lumière. Selon cette nouvelle logique, parallèle à celle des arts performatifs, l'œuvre originale ne serait plus qu'une matrice première, l'origine d'une longue lignée de reproductions qui viendraient l'actualiser. La discussion aurait donc à porter sur les critères de ce qui fait la valeur d'une «bonne» copie.

En complément à ce texte propre à alimenter le débat, on pourra consulter en ligne l'appendice technique qui expose la méthode de duplication numérique employée dans le cas des Noces de Cana ${ }^{4}$. L'essai de Bruno Latour et Adam Lowe paraît par ailleurs simultanément en anglais dans le volume collectif Switching Codes aux Presses de l'Université de Chicago5. Dans cet ouvrage, deux courtes répliques de Charles Bernstein et de Judith Donath accompagnent le texte. Dans la seconde de ces réponses, "Pamphlets, Paintings, and Programs: Faithful Reproduction and Untidy Generativity in the Physical and Digital Domains ", l'auteure souligne la tension qui existe entre physicalité accidentée et organique des objets d'un côté, et netteté lisse du numérique de l'autre. Selon Donath, le passage du temps serait véritablement perceptible sur l'objet physique, peu à peu transformé par les traces mêmes de son histoire. Mais elle suggère que l'on puisse également

3. Voir Peter Greenaway, Veronese, The Wedding at Cana: A Vision by Peter Greenaway, Milan \& New York, Charta Books, 2010.

4. Appendice de Adam Lowe, directeur de Factum Arte, qui a présidé à la reproduction des Noces de Véronèse: www.bruno-latour.fr/articles/article/108-ADAM-FACSIMILES-AL-BL.pdf p. 15-18 (dernière consultation le 22 juin 2011). Voir en complément: Pasquale Gagliardi, Bruno Latour et Pedro Memelsdorff (dir.), Coping with the Past: Creative Perspectives on Conservation and Restoration, Florence, Leo S. Olschki, 2010.

5. Voir Thomas Bartscherer et Roderick Coover, Switching Codes: Thinking Through Digital Technology in the Humanities and in the Arts, Chicago, The University of Chicago Press, 2011. 
concevoir un système dans lequel les copies numériques pourraient être, elles aussi, chaque fois imperceptiblement transformées par le geste de reproduction ${ }^{6}$.

Sans entrer dans le jeu de répliques proposé dans Switching Codes, nous avons de notre côté demandé un commentaire au texte de Latour et Lowe à Johanne Lamoureux qui, dans "Autour de la migration de l'aura: le grand déménagement", nous en offre une lecture d'historienne de l'art. À travers l'analyse de la contribution d'Erwin Panofsky au débat sur les fac-similés d'œuvres, elle rappelle que le jugement de "bonne» ou de "mauvaise» reproduction a toujours constitué pour sa discipline un exercice éclairant, mais dont les critères ont varié au cours du siècle dernier, notamment au chapitre de l'hybridé médiale des reproductions. Cette réponse mentionne aussi l'importance de demeurer vigilant quant aux enjeux soulevés par les possibles usages politiques de la technologie de duplication numérique qui, à leur tour, soulèvent la question difficile et toujours d'actualité du déplacement et de la restitution des ouvres d'art. Souhaitons que l'ouverture de ce débat produise de nouvelles répliques.

6. Voir Judith Donath, ancienne directrice du Sociable Media Group du MIT Media Lab, dans ibid., p. 303. 